\title{
Improvement for performance radio frequency in wireless communication based on impulse signal
}

\author{
Jafaar Fahad A. Rida \\ Department of Information System Computer, Collage of Computer Science and Information Technology, \\ University of Sumer, Iraq.
}

\begin{abstract}
Article Info
Article history:

Received Sep 20, 2019

Revised Nov 23, 2019

Accepted Dec 7, 2019

\section{Keywords:}

Impulse response

Mobile wireless propagation

Radio frequency propagation

Radio multipath

Software filter

ABSTRACT

This research presents the improved performance of mobile wireless communication channel is based on non-line of sight because the mobile propagation channel is very hard to work with direct path between the transmitter and mobile receiver because of city-based area has objects such as rise building, trees, surround structures, and other objects. The mobile wireless spread radio channels have radio propagation mechanisms as a reflection, diffraction, and scattering effect on radio signal as lessening, and distortion or spread time. The most important thing to make real is that the wireless channel is a multipath radio channel. Multipath copies of the same signal with distinct amplitudes, stages and delay times for all multipaths between the transmitter and the receiver. The multipath fading is defined by its impulse response, which includes the facts about the relative time of the recipient's arrival of various indices of multipath additives. The impulse response expresses great characteristics in the time domain such the large bandwidth frequencies and the amplitude output only the value impulse response $\delta(t)$ equal to one when the value of time $t=0$, otherwise, the value impulse response $\delta(\mathrm{t})$ equal to zero in all values of the time. Over a time period reaction, the cellular radio channel could be designed as a linear filter with various impulses.
\end{abstract}

Copyright (ङ 2020 Institute of Advanced Engineering and Science. All rights reserved.

\section{Corresponding Author:}

Jafaar Fahad A. Rida,

Department of Information System Computer,

Collage of Computer Science and Information Technology,

University of Sumer, Dhiqar, Iraq.

Corresponding Emails: j.fahad@uos.edu.iq \& jafaarfahad@yahoo.com

\section{INTRODUCTION}

The remote correspondence framework mainly conveys and receives voice and data using openspace electromagnetic waves or air media that are fundamentally free of cables. This Innovation isn't just transmitting voice yet in addition information (content, number, picture, and video). Today the general population can chat on the cell phones or PDAs are utilized to browse their messages and Web Applications in the meantime [1]. It gets the stock codes particularly when the advanced innovation came the security turns out to be superior to simple innovation. The traffic isn't just giving up to rivalry to voice traffic yet in addition surpassing the voice traffic. The data from sender to the recipient is typically conveyed down over a well-characterized frequency band [2]. This frequency otherwise called the data transfer capacity distributed for remote correspondence is a standout amongst the most prized items and typically unloaded. The remote correspondence isn't one individual. The radio range of frequency is restricted by Government Interchanges Commission (FCC) in The US of America dispersed breaking point transmission capacity recurrence for remote correspondence applications this implies utmost channel and clients to expand the limit is utilized numerous strategies [3-4], for example, different access methods Multiple Access Division (FDMA) is assigned bandwidth into sub-bands, everyone is known as a channel. 
Every client has a special frequency in uplink and downlink. The frequency band is partitioned into channels of equivalent transmission capacity with the end goal that every discussion is completed on various frequencies [5]. The recipient must have a channel, the channel qualities will be imported figuring out what sort of channel data transfer capacities is in effect really utilized. That's going to happen, the situation is actually a ton of frequency cover that can happen due to the treepasses on the other band called obstruction. Here, the straightforward issue is to assign some space between the frequency portions which are known as the guard groups. Guard bands are utilized to restrict crosstalk between channels between adjacent sign spectra. The filter must be very sharp such as hardware filters and software filters as in this research paper is used software filter as Linear Time-Invariant filter based on impulse response gives output one value $\delta(\mathrm{t})=1$ when $\mathrm{t}=0$ otherwise, $\delta(\mathrm{t})=0$. Multiple Access Time Division (TDMA) time slots are specified using the distinct time for different users for communicating a unique time slot for each user. TDMA is used to access various subbands. The subbands are known as carrier frequencies and are designated as various carrier structures for the mobile system using this method. The GSM (Global Mobile Communication System) panEuropean digital system utilizes such a mixture of FDMA and TDMA. The mobile receiver is allocated frequency and time slot sequence performs to the physical TDMA channels framework. The portable receiver sends an information burst in each slot.

The period set for a time slot for a mobile receiver and the number of TDMA channels on the frequency of a carrier. TDMA frame consisted of one sequence of time slots. Qualcomm has created the cellular system based on Code Division Multiple Access (CDMA). Inc. and Telecom Standardization [6-8], Industry Association (TIA) as an Interim Standard (IS-95). This promotes the amount of customers accessible in $1.25 \mathrm{MHz}$ wide channel using the spectrum of direct sequence spread. The CDMA systems are used with the same frequency in each cell, which results in a significant capability enhancement [9]. CDMA's fundamental concept is that distinct codes are used to differentiate between distinct users. Direct Sequence Spread Spectrum (DS-CDMA), Frequency Hopping or Joint Detection CDMA (JD-CDMA) are typically used modulation types. This generates a signal that spreads across a wide bandwidth. For this action, a signal called a spreading code can be used. With a set of codes orthogonal to each other, a signal with confirmed code can be selected in the present presence of many other signals with distinct orthogonal codes. Indeed, a wide range of baseband "signals" with various spreading codes can be modulated onto a similar carrier to empower a wide range of clients to be bolstered. The interference between the signals is minimal by using various orthogonal codes.

The cell phone or mobile phone is the contemporary equivalent of Marconi's wireless telegraph, providing two-way communication between parties, Personal Communication Service (PCS) license in the 900/ $1800 \mathrm{MHz}$ band in early 1995 [10]. There are some advantages in wireless systems such as the first is freedom from wires, the customer can call anytime and anywhere. The second is flexibility as radio waves go through walls, less installation, and cost. On the other side, disadvantages exist like relative lower bandwidth. The more users mean each bandwidth get smaller that means the bandwidth is limited in wireless communication although increase using more applications. It is also known as the ISM band, it is provided to the organization of the medical industry and to experiment on this frequency with a lot of helpful equipment. For example, FM radio which has worked around $80 \mathrm{MHz}-108 \mathrm{MHz}$, the TV broadcast is at $200 \mathrm{MHz}$, the GSM phones work at $900 \mathrm{MHz}$ and $1800 \mathrm{MHz}$, the dual-band phones at two frequencies one for uplink (from user to transmitter) and another downlink (from the transmitter to user).

Global Position Systems (GPS) works at $1.2 \mathrm{GHz}$ and the Bluetooth the poor of the ultra-wideband technology works at $2.4 \mathrm{GHz}$. This translates to get license for frequency for wireless network payment a lot of money for government to get a lot of money by licensing the radio spectrum frequency [11-12]. The mobile wireless propagation improves on the performance of wireless communication systems take these very important points our eyes.

The first point is security for wireless network via digital codes technology for encryption, authentication, privacy, and integrity. A wireless network system requires secure medium access control (MAC) protocol, and the basis of predefined addresses (IP Address). Security is requirements of wireless systems depend on the amount of investment and the characteristics of applications running on the system.

The second is Bandwidth limitations emerge from material and energy physical characteristics. Each physical transmission medium has a limited bandwidth that determines its voice, information, graphics, and video communication effectiveness. Widespread Internet use has boosted government knowledge of the bandwidth of telecommunications because customers and Service suppliers are interested in optimizing the speed and speed of Internet access on computer screens and the user mobile phones use multimedia applications today [13-14].

The third is the mobile wireless communication is difficult work with line of sight (LOS) must be point to point direction without any obstacles between transmitter and receiver this is impossible investigation in mobile environment in urban area has buildings, trees, and other obstacles in the propagation 
environment. The mobile wireless uses non-line of sight (NLOS) that means, the signal propagates from the transmitter to the receiver through various distinct routes owing to barriers and the wireless channel reflectors can be called multi-path channels. The multipath is caused by procedures of reflection, diffraction and dispersion as shown in Figure 1.

The fourth is increase power in the cellular system goes to communicate over large distance with getting attenuated the receiver sensitivity. The transmitted power must increase carefully for two reasons, one the more power is radiating needed equipment efficient hardware to spend more money and other more important thing today is the radiation hazard.

The last is the radio channel model behaves differently in different frequencies that means the wavelength $(\lambda)$ is also different. The wavelength of transmission effects reflected, obstructed or absorbed or diffracted depends on the size of wavelength. The multipath propagation channel is the different paths for the same signal will be different amplitude, phase, and arrival time to reach the receiver as mobile station. The multipath channel can use software filter via impulse response to select strong path with frequency desired when the value of $\delta(t)=1$ when $\mathrm{t}=0$ otherwise, $\delta(t)=0$ by transfer function here impulse response $[15,16]$.

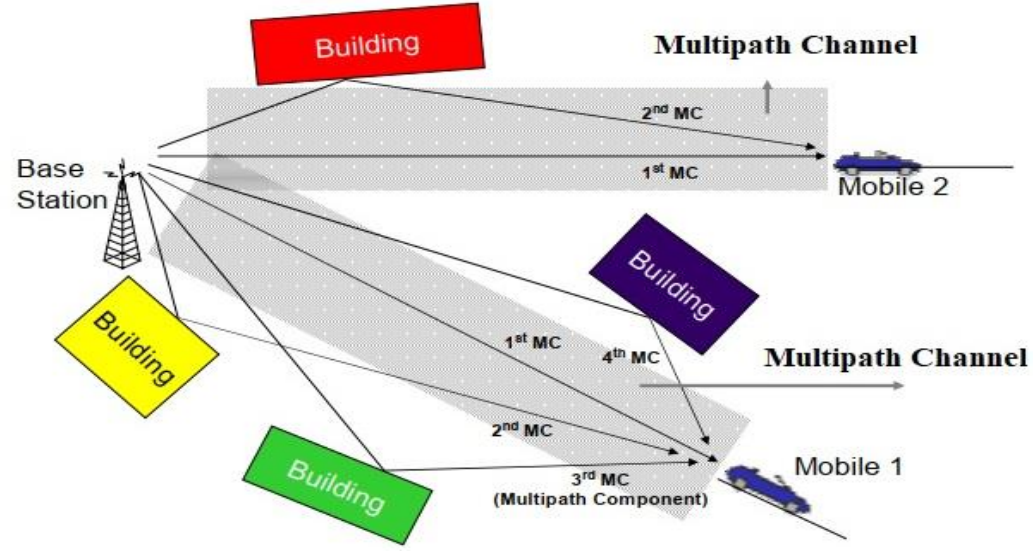

Figure 1. The mobile wireless radio propagation via multipath channel model

\subsection{Multipath Channel Impulse Response}

The impulse function is a very brief pulse for evaluating the dynamics of the scheme. A pulse function in the real world is a pulse that is much shorter than the system's time response. Using the timeslicing method called convolution, the impulse response scheme can be used to decide the system output for each input. Convolution is widely used to introduce digital filters in signal processing. In the time domain, Convolution works the same role as frequency domain multiplication and vice versa. A dynamic system impulse input is described differently based on whether the system is discreet or constant. The impulse response $\delta(t)=1$ has output signal when the value of time $(\mathrm{t}=0)$, and $\delta(t)=0$ when the time all values as given in (1).

$$
\delta(t)=\left\{\begin{array}{cc}
\delta(t)=1 & t=0 \\
\delta(t)=0 & t \neq 0
\end{array}\right.
$$

The impulse signal $\delta\left(t-\tau_{0}\right)=1$ can be output signal when the value of time $\left(t=\tau_{0}\right)$, and $\delta\left(t-\tau_{0}\right)=1$ when the time all values as given in (2).

$$
(t)=\left\{\begin{array}{cc}
\delta\left(t-\tau_{0}\right)=1 & t=\tau_{0} \\
\delta\left(t-\tau_{0}\right)=0 & t \neq \tau_{0}
\end{array}\right.
$$

The impulse signal expresses any signal in time domain in digital communication channels has the narrower the impulse, the wider the bandwidth of the filter with less effect noise and less consumption power [17]. The impulse response is essential to multipath fading channel development and a filter's impulse response in the time domain is proportional to the frequency domain filter bandwidth. It is possible to present any signal as a total of scaled and delayed impulses such as given in (3). 


$$
x(t)=\sum_{\tau_{k}=-\infty}^{\infty} x\left(\tau_{k}\right) \delta\left(t-\tau_{k}\right)
$$

The impulse reaction of the system can characterize a signal handling scheme [16]. The impulse reaction is indicated as $\mathrm{h}(\mathrm{t})$ as informed in Figure 2.

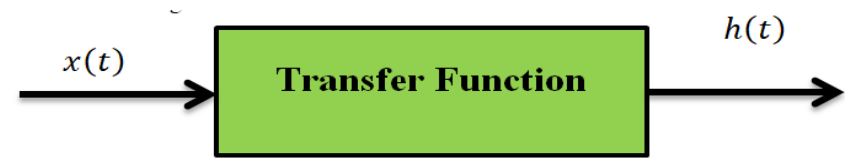

Figure 2. Signal processing system can be characterized by the impulse response of the system

The fundamental problem of signal processing is how to relate a system's input and output signals. The convolution uses a system's impulse response. If the input signal is presented as a sum of impulses, the system's response to different impulses can be formed separately. The reaction to the input signal can then be created as a sum of the various impulses. There are three alternative approaches to a system's impulse response, it can be evaluated if the system is a physical system (e.g. communication channel), it can be calculated if the mathematical representation of the system is known, and it can be constructed using suitable filter design software according to specific applications. A versatile radio channel has appeared as a direct channel with a period changing drive reaction, where the time ranges is because of space gathering. The impulse response is an extremely helpful channel representation as it can be used to predict and consider displaying a few distinctive mobile wireless frameworks and transmission bandwidths for a specific mobile channel scenario. The small differences in a portable radio signal could be directly linked to the mobile radio channel's impulse response. The impulse response is a broadband representation of the channel and contains all important data to reactivate or break down nearly any radio transmission via the channel. The portable channel can be modeled with a time-varying impulse response as a linear filter. The multipath channels change due to the position of the phone and the multi-path fading is distinguished by the impulse response of the channel that contains relative time data, signal power and signal phase $[1,18]$.

\section{RESEARCH METHOD}

A great many people know about various versatile radio correspondence frameworks utilized in regular day to day existence. Examples of radio communication systems include garage door openers, home entertainment remote controllers, cordless telephones, handheld walkie-talkies, pagers and cell phones. However, the costs, complexity, efficiency improvements and kinds of services provided by each of these mobile technologies differ widely. The term mobile has historically been used to classify any radio terminal that could be moved during operation. More recently, the term mobile is used to describe a radio terminal linked to a high-speed mobile platform such as a mobile phone in a fast-moving vehicle, while the term mobile describes a hand-held radio terminal that can be used as a walkie-talkie or cordless telephone inside a home by someone at walking speed. Multipath on the radio channel increases the impact of small scale. For example, the three most significant impacts are fast changes in sign quality over a short range or interim period, uneven recurrence equilibrium owing to fluctuating Doppler movements on the distinct multi-path flag, and multi-path delays in moment scattering (echoes). Fading occurs in advanced metropolitan areas due to the height of the mobile antennas less than the height of the surrounding buildings. There is no direct route between the transmitter and the receiver unit, it requires multipath depending on the ground and surrounding structures reflections. The incoming radio waves come with various delays in propagation from distinct directions [19]. The signal that the phone receives in space at any time can consist of a huge number of plane waves that have distributed amplitudes, stages, and entry edges arbitrarily. If items in the radio channel are static and motion is regarded solely because of the mobile, blurring at that stage is merely a spatial marvel.

The collector considers the spatial varieties of the following sign as temporary variants as they travel through the multipath domain. Because of the constructive and dangerous effect of multitrack waves summing up at different spatial points, a quickly moving reception can pass a few fades in a short time. Due to the comparative movement between the portable and base station, each multi-path wave experiences an apparent shift frequency. The movement in received signal frequency owing to movement is known as the Doppler's movement and is legally linked to mobile movement velocity and direction with regard to the movement of the received multipath signal. The proliferation of multipaths that are in the channel due to the proximity of reflecting objects and scatterers allows a constantly changing situation that disseminates the 
vitality of the signal in abundance, phase and moment. The uneven phase and amplitudes of the unique multipath components trigger changes in the quality of the signs, in this way a little or both twisting of the blurring signal. Multipath spread regularly extends the time required to reach the beneficiary by the baseband segment of the sign, which can cause sign spread due to inter-symbol obstruction. With the impulse response of the versatile radio channel, the small varieties of a portable radio sign can be legally recognized. The impulse response is a depiction of wideband channels and includes all the information needed to recreate or break down any sort of channel radio transmission. This arises from the possibility of demonstrating a portable radio channel as a straight channel with a period moving impulse response, where the time range is due to collector motion in space. The filtering nature of the channel is caused by the summation of the amplitudes and delays of different arriving signals at any time. Here, the mobile radio propagation channel improves multipath propagation channel with impulse response occurs software filter which is given the output signal when the value of time $t=0$ otherwise, it is not an output signal. The impulse response is linked to the bandwidth to differentiate between the required signal and other signals such as noise [20-23].

\subsection{Basics of Radio Propagation}

The antenna can be defined as an electrical conductor or conductor system used for the collection of electromagnetic energy or electromagnetic energy. The receiving device transforms electromagnetic radio recurrence into electromagnetic vitality and emanates into the extensive condition (environment, space, and water) for the transmission of a signal. To receive a signal, electromagnetic vitality that affects the receiving apparatus is transformed into electrical energy of radio frequency and nourished into the recipient. In two Way correspondence, a comparable radio wire can be used for transmitting and receiving on a regular basis. The transmission route between the transmitter and the receiver can either be easy to see or blocked by structures, hills, and folds. This is conceivable considering that any radio wire transfers energy from the encompassing position to its input receiving terminals with similar effects from the output transmitter terminals to bearings. An antenna signal travels one of three routes, such as surface waves, sky waves and a direct trajectory.

The propagation of the ground wave follows more or less the contour of the earth and can spread significant distances over the visual horizon. This impact can be discovered up to about $2 \mathrm{MHz}$ in frequency. This frequency range for electromagnetic waves is dispersed through the atmosphere, meaning that they cannot penetrate the atmosphere. AM radio is the best known case for ground wave correspondence. Sky wave propagation is used for $\mathrm{BBC}$ and Voice of America's amateur radio, CB radio and international broadcasts. The propagation of the sky wave is expressed as a signal from the floor-the receiving wire is reflected from the ionized layer of the withdrawal to earth from the upper atmosphere (ionosphere). An enormous amount of kilometers from the transmitter can be captured with this spread mode. The line of sight is above $30 \mathrm{MHz}$, the mode of propagation of the ground wave or the sky wave does not work and communication must be by line. Refraction occurs because an electromagnetic wave's velocity is an aspect of the medium's density through which it travels. In vacuum waves such as light or a radio wave, electromagnetic waves travel at approximately $3 * 10^{8} \mathrm{~m} / \mathrm{s}$. Electromagnetic waves travel at speeds slower than the speed of light in the air, glass, and other transparent media. The size of antenna depends on wavelength $(\lambda)$ where the frequency increases become the wavelength decrease that performs the size of antenna becomes small. The radio wave can be penetrated the wall in buildings, easy generated and work properly with safe environment. Due to distinct transmission impairments, the signal acquired with any communication system will differ from the signal transmitted. Deterrence and distortion, free space loss, noise, atmospheric absorption, and reflection are the most important impairments [24-26].

\subsection{Basics of Antenna}

A radio wire will emanate power in any way, but usually does not do the same thing in any way. A popular way to characterize the output of an antenna is the radiation pattern, which is a graphic representation of the radiation properties of an antenna as characteristics of space coordinates. A romanticized reception device known as the isotropic radio wire provides the least challenging illustration. An isotropic receiving wire is a space point that similarly emanates control every way. The actual radiation layout for the isotropic radio wire is a circle with the inside receiving wire. Radiation patterns, however, are almost always shown as a two-dimensional cross section of the three-dimensional pattern. The distance between the antenna and each phase of the radiation pattern is proportional to the energy radiated from the antenna in that direction. The radiation pattern provides a convenient way to determine an antenna beamwidth, a common measure of the direction of an antenna [27]. Also known as the half-power beamwidth, the beam width is the angle in which the radiated energy of the antennas is at least half of what it is in the most preferred direction. The longest part of the pattern shows the best way to get. The Hertz dipole antenna and the vertical or Marconi quarter-wave antenna are two of the most basic and simplest antennas.

Improvement for performance radio frequency in wireless communication based... (Jafaar Fahad A. Rida) 
The parabolic reflector antenna used in terrestrial microwave and satellite applications is a significant form of antenna. The locus of all points equidistant from a fixed line is a parabola. The directionality of an antenna can be measured by antenna gain. Antenna gain is described as the energy output of the ideal omnidirectional antenna (isotropic antenna) in a specific direction compared to that generated in any direction. An antenna gain idea is the efficient region of the antenna [28]. The physical size and shape of the antenna are related to the effective area. The antenna gain relationship with an effective area is given by (4).

$$
G=\frac{4 \pi A_{e}}{\lambda^{2}}
$$

Where antenna gains $(\mathrm{G})$, efficient region (A e), carrier frequency (f), light speed $\left(c=3 * 10^{8} \mathrm{~m} / \mathrm{s}\right.$, and carrier wavelength $(\lambda)$. The free space energy obtained is provided by the free space on Friis is presented $(5)$.

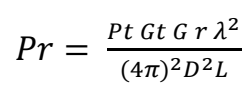

Where transmitted energy (Pt), antenna gain in a transmitter $(\mathrm{Gt})$, antenna gain in a receiver $(\mathrm{Gr})$, wavelength $(\lambda)=\mathrm{c} / \mathrm{f}$., distance between transmitter and receiver $(\mathrm{d})$, and route losses (L). The effective isotropic radiate power (EIRP) is defined as EIRP=Pt $*$ Gt. The loss of track is the attenuation of the signal as a favorable quality is measured by decibel as given by (6).

$$
P L=-10 \log _{10} \frac{P t}{P r}=-\log _{10} \frac{\lambda^{2}}{(4 \pi d)^{2}}
$$

\subsection{Mechanisms for Mobile Radio Transmission}

The mobile communication system has the three fundamental processes of propagation are reflection, diffraction, and dispersion. The buildings behind the spread of electromagnetic waves, however, can mostly be ascribed to reflection, diffraction and dispersion. Most cell radio frameworks work in urban regions where there is no immediate link between the transmitter and the collector and where the proximity of elevated buildings causes serious diffraction misfortune [5].

The electromagnetic waves travel along with distinct ways of moving lengths due to countless reflections from distinct products. Cooperation between these waves creates multipath blurring in a given region, and wave characteristics decrease as a separation between increments of the transmitter and the collector. Spread models have usually focused around predicting the ordinary signal quality in certain good ways from the transmitter, just like the fluctuation of the sign quality in the immediate spatial vicinity to a specific place. Propagation models predicting average signal power for an arbitrary transmitter-receiver (T-R) separation range is useful in estimating the radio coverage area of a transmitter as large-scale propagation models since they characterize signal authority over large $\mathrm{T}-\mathrm{R}$ separation ranges (several hundred or thousand meters). Again, spreading models depicting the fast variations of the received sign quality over brief travel separations (a few wavelengths) or a short time (in the order of seconds) are referred to as tiny scale or fading models [29]. The signal strength immediately acquired can fluctuate rapidly as mobile moves over very small distances, leading in small-scale fading. Reflection takes place when a spreading electromagnetic wave invades an object which, contrasting with the wavelength of the spreading wave, has enormous steps. There are reflections from outside the earth and from constructions and divisions. Diffraction occurs when a surface with sharp anomalies (edges) hinders the radio route between the receiver and the beneficiary. The optional waves resulting from the blocking surface are available throughout the room and even behind the snag so that waves can rise to twist around the impediment, despite the fact that there is no visible pathway between transmitter and recipient as shown in Figure 3. At high frequencies, diffraction, similar to reflection, relies on the geometry of the object just as the sufficiency, stage, and polarization of the episode wave for diffraction purposes Dispersing occurs when the wave travel consists of articles with measurements that are little contrasted with the wavelength and where the number of obstructions per unit volume is enormous. Unpleasant surfaces, small objects or various anomalies in the channel create dispersed waves. Foliage, road signs and lamp posts cause dispersion in a mobile communication scheme in practice $[30,31]$. 


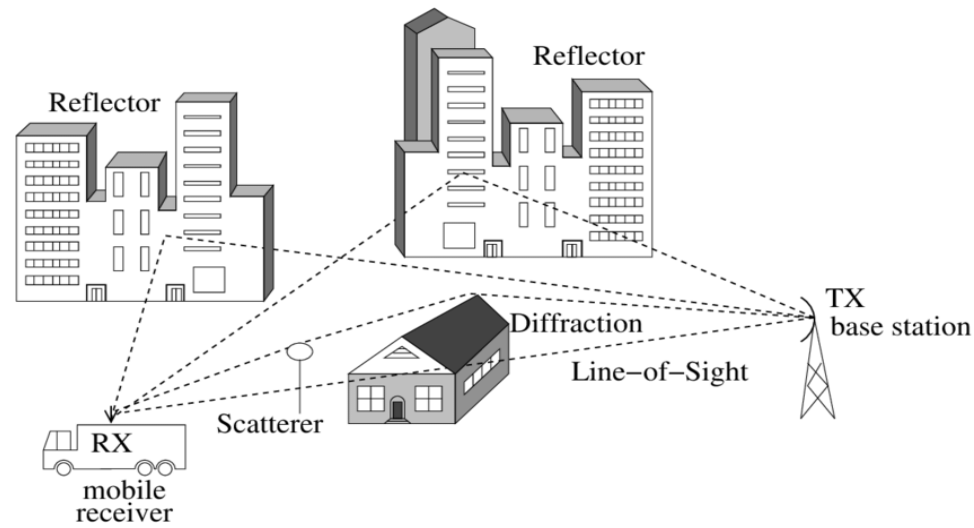

Figure 3. The propagation mechanism basics are reflection, diffraction and scattering in mobile radio propagation channel

\subsection{Small Models of Propagation}

Fading of small scale used to depict the rapid vacillation of the sufficiency of a radio sign over a short time frame or separation of travel, thus ignoring the enormous loss of path impacts. Fading is caused by interference between at least two transmitted sign renditions that touch the basis of the recipient on slightly distinct occasions. These waves are called multipath waves, join the receiver radio cable to provide a corresponding sign that can vary extensively in abundance and phase, depending on the energy and relative distribution time of the waves and the signal bandwidth transmitted [32-33]. The relative movement between the base station and the mobile result in an irregular frequency balance owing to distinct Doppler shifts on each of the multitrack segments, the Doppler shift will be positive or negative depending on whether the mobile receiver moves to or from the base station. If objects in the radio channel are in motion, they result in a time-varying shift from Doppler to multi-path components. In the case that the comprehensive papers move at a more remarkable pace than the versatile, this effect will overwhelm the small-scale fading at that stage. If the transmitted radio signal bandwidth is higher than the multipath channel bandwidth, the received signal will be distorted but the received signal strength will not fade much across a local region, the channel bandwidth can be quantified by the coherence bandwidth associated with the channel's particular multipath structure. The bandwidth of coherence is a percentage of the most severe frequency comparison for which sign is still inadequate. On the off chance that when contrasted with the channel, the transmitted sign has a limited bandwidth, the sign sufficiency will change rapidly, but the sign will not be misfigured in time [34-36].

\subsection{Impulse Response of Multipath Channel}

Signal propagation between transmitter and receiver can be conceptualized in mobile radio by integrating the concept of the mobile radio channel's impulse reaction that causes the signal to filter [17]. The channel's impulse response should be time-invariant the impulse response of the baseband, $h(t)$ is represented as (7).

$$
h(t)=\sum_{i=1}^{N} \alpha i e^{\theta i} \delta(t-\tau i)
$$

Where $\alpha i$ is Amplitude of voltage of the $i^{t h}$ Signal arrival, $\boldsymbol{\theta} \boldsymbol{i}$ is the phase shift of the $i^{\text {th }}$ arriving signal and $\tau i$ is the time delay of the $i^{t h}$ arriving signal. The received signal is generally a sequence of delayed, phase-shifted, attenuated versions of the signal transmitted. The $\mathrm{h}(\mathrm{t})$ parameters can be evaluated directly using wideband channel sounding methods. The Impulse response of the versatile radio channel makes it possible to legally recognize the small varieties of a portable radio signal. The impulse response is a portrait of a wideband channel and includes all the information needed to reactivate or investigate any sort of channel radio transmission. This results from the option of showing a portable radio channel as a direct channel with a time-shifting impulse response, in which the time range should move the receiver in space [37]. The filtering nature of the channel is caused by the summation at any time of the amplitudes and delays of the multiple. The impulse response is a useful channel depiction for predicting and looking at the exhibition of a broad spectrum of mobile wireless frameworks. The output system expresses convolution between the $\mathrm{x}(\mathrm{t})$ input signal and the $\mathrm{h}(\mathrm{t})$ transfer or impulse reaction function as specified by (8) and (9). 


$$
y(t)=\alpha_{0} e^{\emptyset 0} x\left(t-\tau_{0}\right)+\alpha_{1} e^{\emptyset 1} x\left(t-\tau_{1}\right)+\alpha_{2} e^{\emptyset 2} x\left(t-\tau_{2}\right)+\cdots+\alpha_{k} e^{\emptyset k} x\left(t-\tau_{k}\right)
$$

Where $\left(t-\tau_{0}\right)=A \delta\left(t-\tau_{0}\right)$, the direct path is $\alpha_{0} e^{\emptyset 0} x\left(t-\tau_{0}\right)$ between transmitter and receiver, the first multipath is $\alpha_{1} e^{\emptyset 1} x\left(t-\tau_{1}\right)$, the second multipath is $\alpha_{2} e^{\emptyset 2} x\left(t-\tau_{2}\right)$, and other number $n^{t h}$ multipath is $\alpha_{k} e^{\emptyset k} x\left(t-\tau_{k}\right)$. It is possible to change the $\mathrm{x}(\mathrm{t})$ input signal and the $\mathrm{h}(\mathrm{t})$ impulse reaction to form (9).

$$
y(t)=\sum_{k=0}^{N} \alpha_{k} e^{\emptyset k} \delta\left(t-\tau_{k}\right)=\sum_{k=0}^{N} h\left(\tau_{k}\right) \delta\left(t-\tau_{k}\right)=h(t) * \delta(t)
$$

Using convolution, the reaction of an LTI system to an arbitrary input signal can be determined. Signals added at the input in an additive scheme generate signals added at the output. The linear timeinvariant channel's impulse response should depend on the position of the receiver due to the distinct multipath waves with propagation delays that differ across separate spatial places of the receiver. [38].

\section{RESULTS AND ANALYSIS}

The mobile wireless communication channel is based on indirect path because the mobile channel of propagation is very difficult work with direct path between the transmitter station and cell phone station because of urban area has objects and obstacles such as rise building, trees, surround structures, and other objects. The mobile wireless propagation radio channels have radio propagation mechanisms as a reflection, diffraction, and scattering effect on radio signal as attenuation, and distortion or spread time. The most important thing to realize is that the wireless channel is a multipath radio channel, the radio waves the emanate, do not use a single route from the transmitter to achieve the receiver routes. In reality, there are several routes in the radio channel called multipaths that cause fast fluctuation of the signal amplitude called tiny scale fading is a temporary impact and creates destructive interference of two or more versions of the transmitted cell signal that arrives at the receiver is slightly distinct times or may be constructive. These paths arrival in receiver have different amplitude of the signal, different phase, and different time arrival sum of paths with take direction for each other carefully may be constructive or destructive. The multipath copies of the same signal for all multipaths between the transmitter and the receiver with different amplitudes, phases, and delay times. These will come from different directions, the receiver receives signals from a certain most likely this strongest reflected signal and cutoff other frequency for other signals. The multipath fading is defined by its impulse response, which involves data on the relative moment of the arrival at the receiver of various multipath component signals. The impulse response expresses great characteristics in time domain such the large bandwidth frequencies and the amplitude output only the value impulse response $\delta(t)$ equal to one when the value of time $t=0$, otherwise, the value impulse response $\delta(t)$ equal to zero in all values of time. The impulse response can use filter or software filter with cutoff frequency for desired signal. By convolution the input signal to the impulse reaction signal which gives output signal when $\mathrm{t}=0$ or $\mathrm{t}=\mathrm{t} 0$. The filter's impulse response can be calculated by taking the inverse discrete Fourier frequency response transformation. Convolution can be used to determine the reaction of the Linear Time Invariant (LTI) scheme to an arbitrary input signal. A change in the input creates a comparable change in the output in a time invariant scheme. This implies that the system's characteristics remain continuous over time. It is possible to demonstrate the mobile radio channel as a direct channel with a period shifting impulse reaction. We can truly break down a channel at any ideal frequency band. A multipath channel's impulse reaction includes all the data needed to boost the channel and evaluate the channel $[1,5,6,16,30,36-39]$. Depending on the distance between the transmitter and the receiver, the received energy will combine distinct incoming signals with distinct propagation delay moment with constant velocity for moving the mobile receiver as given (10).

$$
\mathrm{t}=\mathrm{d} / \mathrm{v}
$$

Where $t$ is due exclusively to the movement of the receiver in space, $d$ is the receiver moving along the floor depending on distance between the sender and receiver radio channel, and $\mathrm{v}$ is constant velocity

In Figure 4 watches the variation times are expanding when the separations between the transmitter and the portable beneficiary are expanding. The principal bend (red shading) is spoken to the variation times with separations for movement versatile position from the transmitter station with consistent speed for portable moves is $\mathrm{v}=10 \mathrm{mk} / \mathrm{h}$, the second curve (green shading) with steady velocity is $\mathrm{v}=50 \mathrm{~km} / \mathrm{h}$ and the last bend (blue shading) with steady velocity is $100 \mathrm{~km} / \mathrm{h}$. These bends are demonstrated the variation times for moving portable when the versatile position builds separations with velocity is steady. 


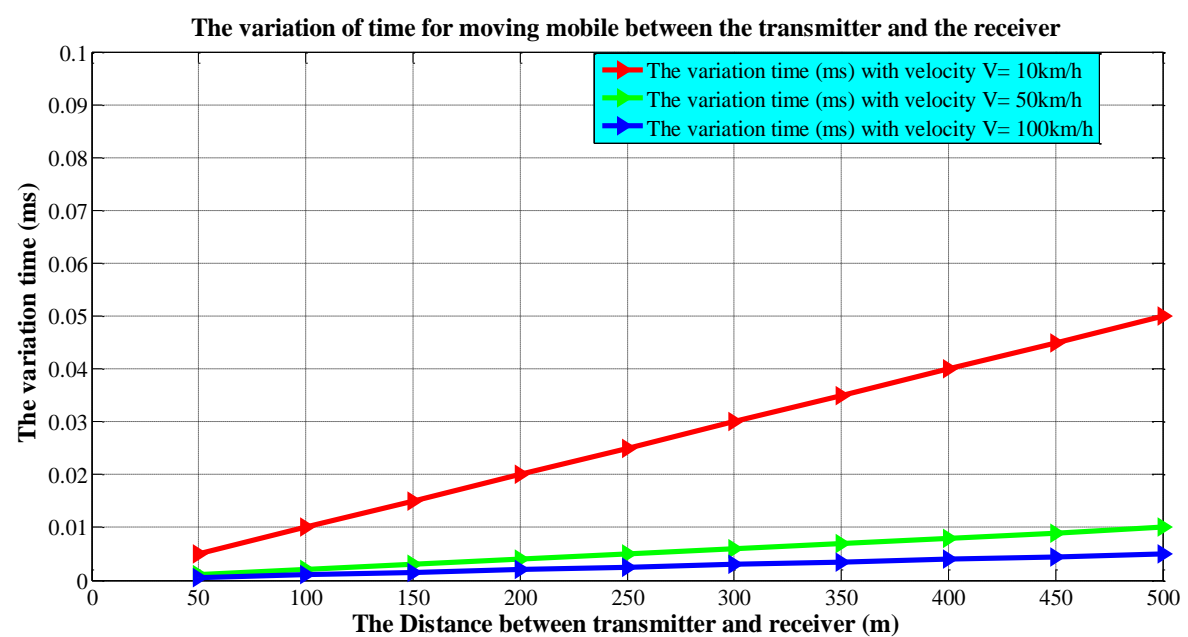

Figure 4. The variation of time for moving mobile receiver from the transmitter

The impulse response multipath radio spread channel for this framework is given by condition (11).

$$
h(t)=h 0+h 1+h 2+\cdots+h N-1
$$

Where $\mathrm{h}$ is move capacity is relying upon separation between the transmitter and the versatile recipient position is given by (12).

$$
h=\frac{\lambda}{d} e^{j \frac{2 \pi d}{\lambda}}
$$

Compensation (12) in (11) as shown in (13).

$$
h(t)==\frac{\lambda}{d 1} e^{j \frac{2 \pi d 1}{\lambda}}+=\frac{\lambda}{d 2} e^{j \frac{2 \pi d 2}{\lambda}}+=\frac{\lambda}{d 3} e^{j \frac{2 \pi d 3}{\lambda}}+\cdots+=\frac{\lambda}{d N-1} e^{j \frac{2 \pi d N-1}{\lambda}}
$$

Where $\lambda$ is wavelength for this framework has the carrier frequency is $f=900 \mathrm{MHz}$ and the separation is $d$ between the transmitter and the mobile receiver.

The results depict the features of the time domain impulse reaction used in the multipath radio channel. The signal desired can pass and reject other undesired signals via impulse response channel bandpass filter as a linear time-invariant filter channel as shown in Figure 5.

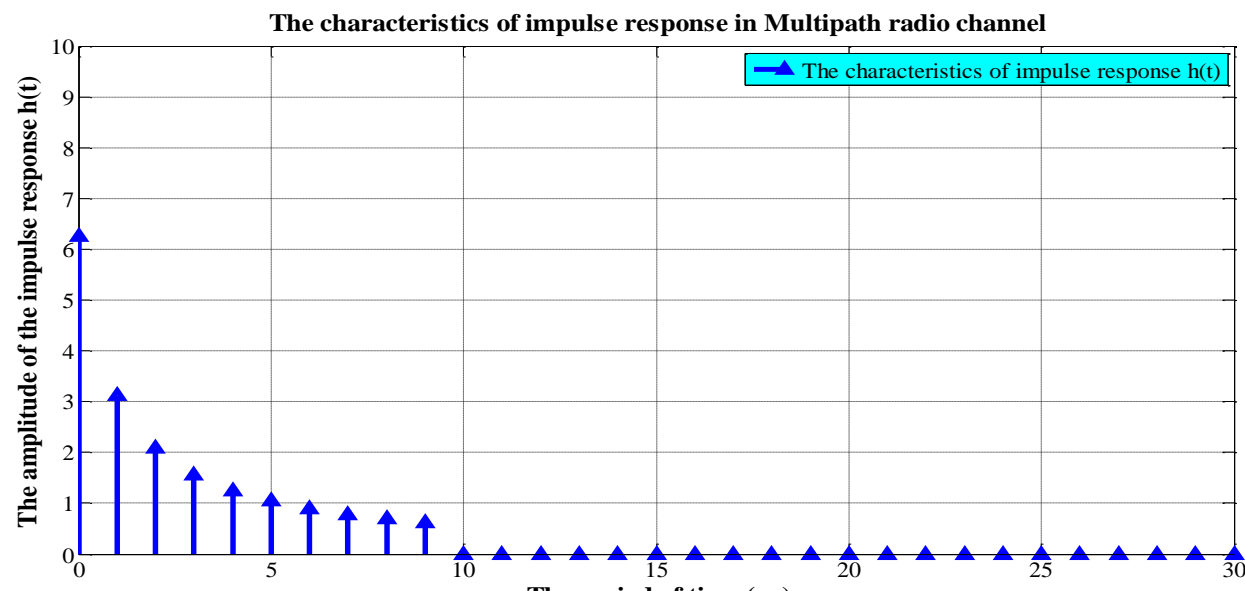

The period of time (us)

Figure 5. The characteristics of impulse response in multipath radio channel 
The amplitude of the impulse reaction $\mathrm{h}(\mathrm{t})$ is reduced by the range for the position of the portable receiver. Figure 6 demonstrates frequency response characteristics of impulse response in multi-path radio channels in the frequency domain using discrete Fourier shift (DFT), the magnitude of the frequency response as the passband channel and the reduction of the signal due to the fading of the channel.

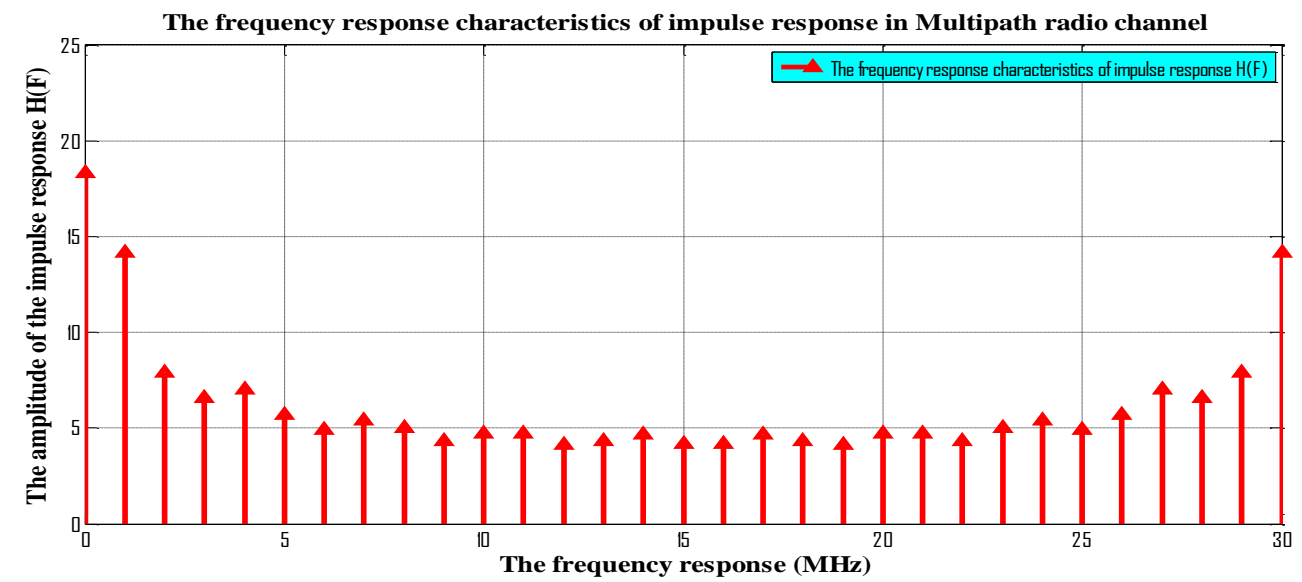

Figure 6. The frequency response characteristics of impulse response in multipath radio channel

The phase response characteristics for impulse response characteristics is depending on distance multipath radio channel to reach at the mobile receiver in fixed time and the phase signal after discrete Fourier transform output as illustrated in Figure 7.

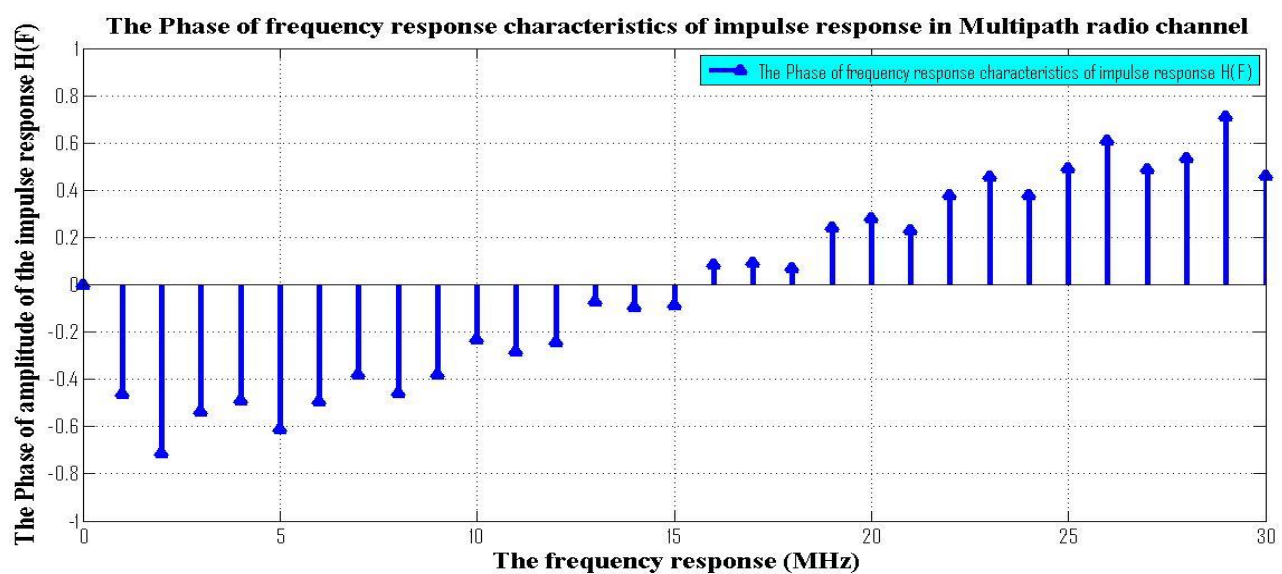

Figure 7. The phase response characteristics of impulse response in multipath radio channel

The output scheme is convolution between the $\mathrm{x}(\mathrm{t})$ signal and the $\mathrm{h}(\mathrm{t})$ transfer function is provided the $y(t)$ output as shown in (9). The results are obtained passband signal for desired for multipath components decrease amplitude of the signal with delay time arrival multipath at the mobile position. Here, we see in Figure 8 illustrates the direct path or line of sight into the mobile receiver is the best path arrival receiver when $\mathrm{t} 0=0$, the first is reflected path or non-line of sight but it has a delay time and the second reflected path has the time delay more than the first path as well as the amplitude for the second path less than the first path, the same conditions for other multipath channels in time domain. The sum of multipath channels may be constructive or destructive with different phases or directions. Intersymbol interference (ISI) is symbols arriving a large delay time or late paths interfere with following symbols. Channel fading is symbols arriving along with different paths sum up destructively this problem in the wideband channel. The narrow band is approximated by a single path channel. 


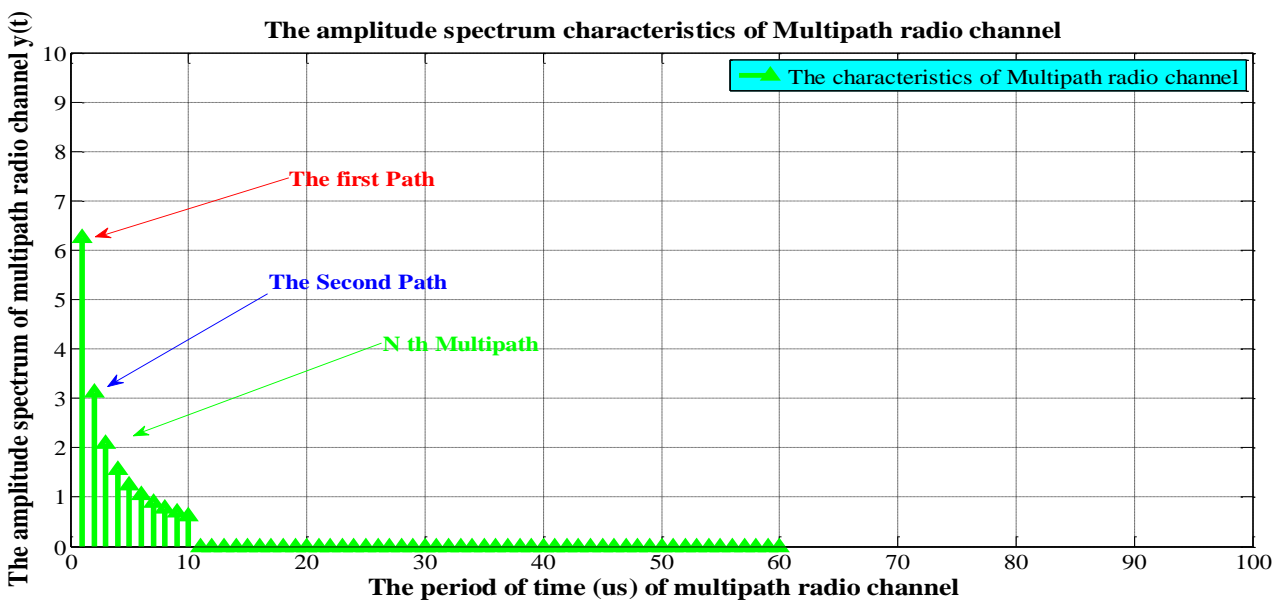

Figure 8. The amplitude spectrum characteristics of multipath radio channel pass band filter in time domain

The outcomes amplitude of the output signal convolution in frequency response pass band filter the output signal amplitude is decreasing in middle because of fading channel or destructive multipath channels. The discrete transformation of Fourier receives frequency response for the output signal as presented in Figure 9.

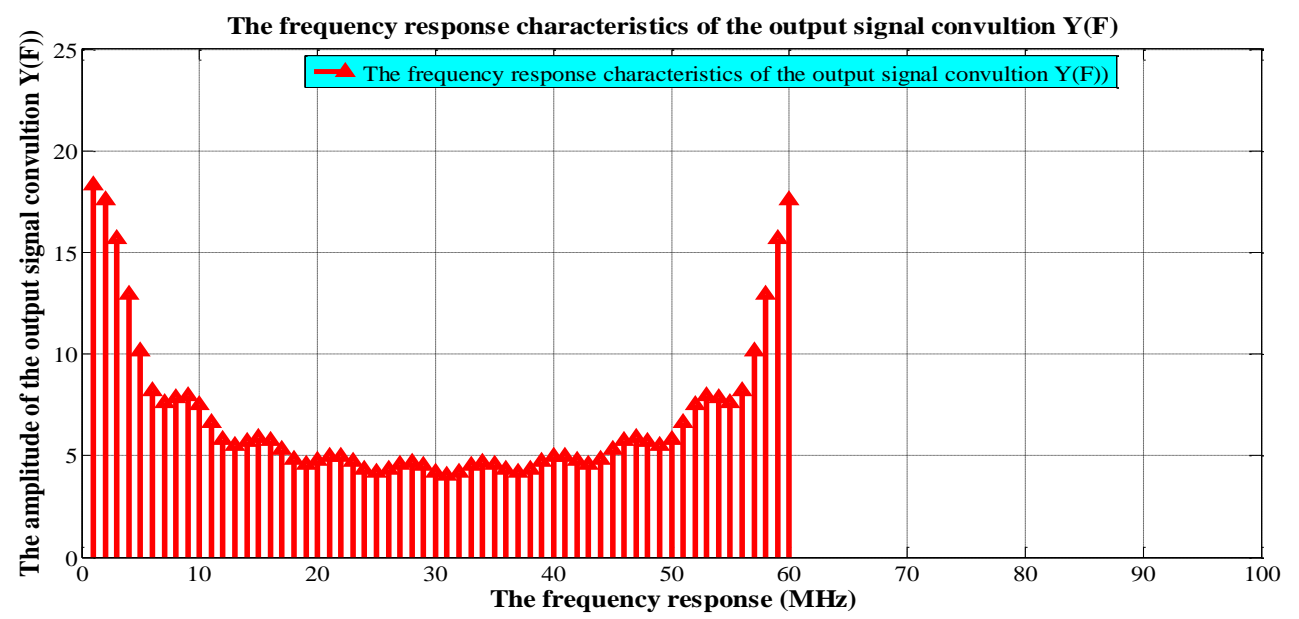

Figure 9. The frequency response of the output signal convolution pass band filter in frequency domain by (14).

The amplitude spectrum of multipath radio channel as baseband filter The time domain is specified

$$
h_{b}(t)=\frac{1}{2} h(t)
$$

Where $h_{b}(t)$ is impulse response in multipath channel for base band filter as indicated Figure 10 observes convolution between signal input and response impulse for base band in time domain, the outcomes are gradual decrease paths depending the stronger signal and delay time to arrive the mobile unit. In Figure 11 shows the frequency response for the output signal via discrtete fourier transform. The amplitude value the output signal in pass band filter is larger than the base band signal, the system can convert between each other he software filter can be used in multipath radio channel as a bandpass filter and baseband filter based on impulse response signal is a linear time-invariant filter is more efficient than hardware filter is difficult available components and little sensitivity signal input. 


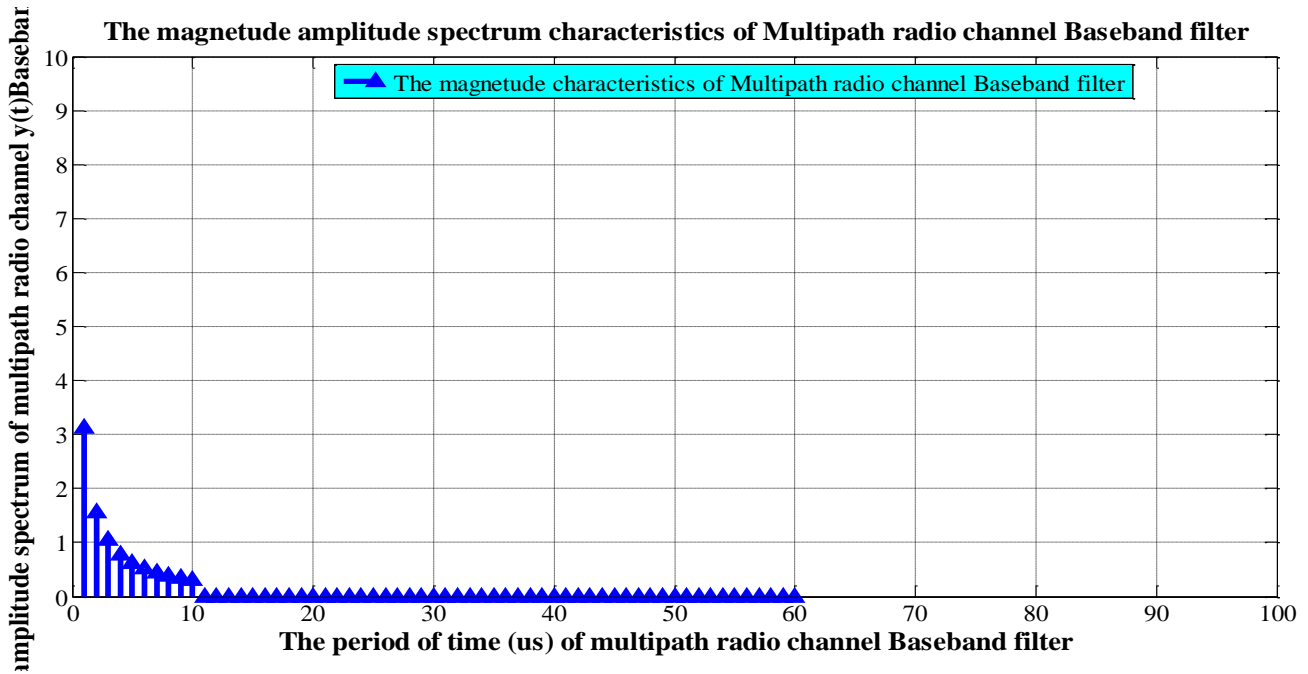

Figure 10. The amplitude spectrum characteristics of multipath radio channel base band filter in time domain

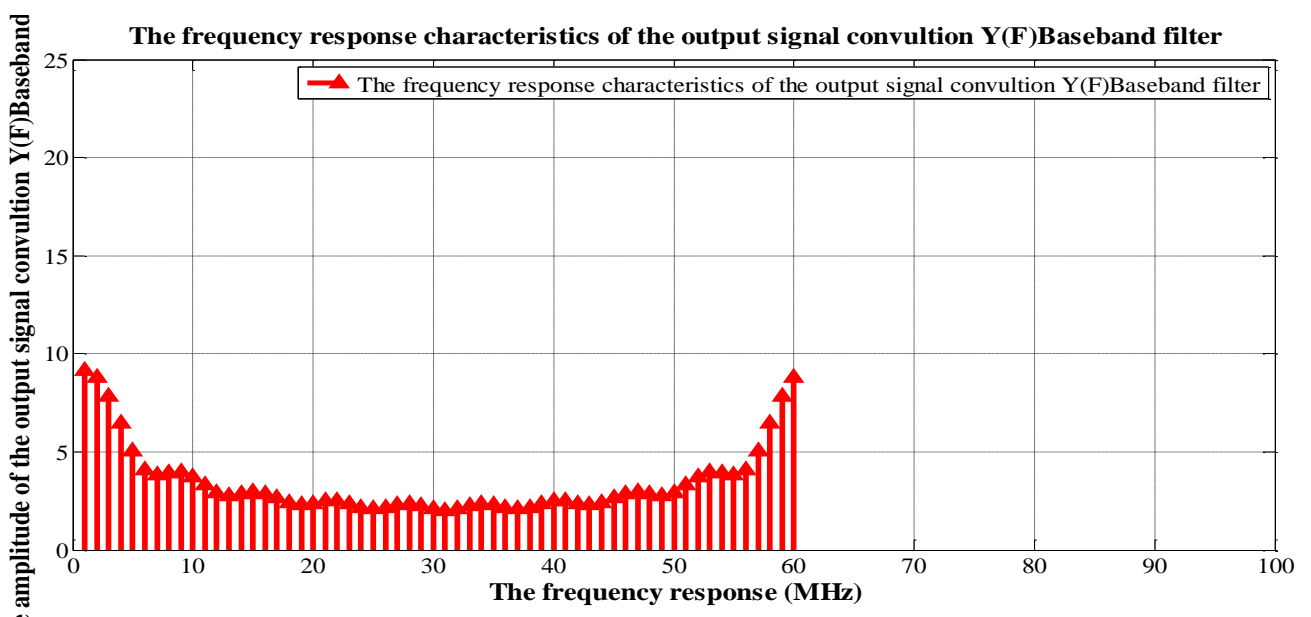

Figure 11. The frequency response of the output signal convolution pass $b$ and filter in frequency domain

\section{CONCLUSION}

A mobile radio channel is shown as a linear filter with a time-changing momentum reaction, where the time range is due to the receiver's movement in space. The impulse reaction is a valuable portrayal of the channel as it is utilized to anticipate and look at the presentation of a wide range of mobile wireless frameworks and transmission data transfer capacities for a specific mobile channel condition. The small varieties of a portable radio sign can be legally identified with the impulse response of the portable radio channel. The impulse response is a broadband channel representation and contains all important data to reproduce or break down a channel radio transmission. The portable channel can be modeled with a timevarying impulse response as a linear filter. The multipath channels are changing because of the position of the mobile and multipath fading is defined by a channel reaction with the relative moment, signal energy and signal stage data. Because of the constructive and harmful impact of multitrack waves summing up at distinct spatial locations, a rapidly moving collector can spend a few hours in a short time frame This arises from the possibility of demonstrating a mobile radio channel as a direct channel with a period fluctuating impulse response, where the time range is supposed to move the receiver in space. Mobile radio propagation channel improves multipath propagation channel with impulse response occurs software filter. The filter's impulse response can be calculated by taking the frequency response's inverse discrete Fourier transformation. Convolution can be used to determine the reaction of the Linear Time Invariant (LTI) scheme to an arbitrary input signal. The sum of the multipath channels can be constructive or destructive with different phases or directions. Intersymbol interference (ISI) is symbols arriving a large delay time or late paths interfere with 
following symbols. The software filter can be used in multipath radio channel as a bandpass filter and baseband filter based on impulse response signal is a linear time-invariant filter is more efficient than hardware filter is difficult available components and little sensitivity signal input.

Ethical clearance: People identified as potential research participants because of their status as relatives or carers of patient's research participants by virtue of their professional role in the university and departments.

Source of funding: Self-Funding

Conflict of interests: The authors declare there is no conflict interests.

\section{REFERENCES}

[1] Theodore S. Rapport (2002)" Wireless Communications Principles and Practice " $2^{\text {nd }}$ edition, Pearson. pp 69 - 197.

[2] Aftab Ahmad (2005) "Wireless and Mobile Data Network" Wiley - Interscience. A John and Sons, INC. Publication, pp 197-273.

[3] Lou Frenzel (2012) "Understanding Solutions For The Crowded Electromagnetic Frequency Spectrum", a contributing Communications Technology Editor for Electronic Design.

[4] Health Canada (2015) "Limits of Human Exposure to Radio frequency Electromagnetic Energy in The Frequency Range From 3 KHz To 300 GHz" Her Majesty the Queen in Right of Canada, as represented by the Minister of Health, pp $1-14$.

[5] William Stallings (2005) "Wireless Communications and Networks", 2 nd edition, Pearson, Prentice Hall, pp 264-314.

[6] Andrea Goldsmith (2005) “Wireless Communications” Cambridge University Press, pp 64 - 94.

[7] Frenzel, Louis E. (2008) "Principles of Electronic Communication Systems", $3{ }^{\text {rd }}$ Edition, McGraw Hill pp 330 - 380.

[8] Gibson, Jerry D. (1997) “The Communications Handbook”, CRC Press, pp 108 - 115.

[9] Upamanyu Madhow (2008)" Fundamentals of Digital Communication”, Cambridge University Press, pp379 - 453.

[10] Vijay Garg,(2000) "IS-95 CDMA and cdma2000”, Prentice Hall, New Jersey.

[11] Vijay K. Garg (1999)" Principles and Applications of GSM"6 $6^{\text {th }}$ edition, Pearson Education Inc.

[12] George Kizer (2013)" Digital Microwave Communication Engineering Point to Point Microwave System” John Wiley \& Sons, Inc., Hoboken, New Jersey, Canada pp 20 -217.

[13] N. K. Ratha, J. H. Connell, and R. M. Bolle (2010) "Enhancing security and privacy in biometrics-based authentication systems," IBM Syst. J. pp 62-69

[14] Georgios Kambourakis, Felix Gomez Marmol, and Guojan Wang(2018) “ Security and Privacy in Wireless and Mobile Networks" $1^{\text {st }}$ edition, MDPS, pp 1-50.

[15] C. L. Holloway, G. Koepke, D. Camell, K. A. Remley, D. F. Williams, S. Schima, S. Canales, and D. T. Tamura (2006) "Propagation and Detection of Radio Signals before During and after Implosion a Large Convention Center" NIST Technical Note 1542, Boulder, CO.

[16] T.S. Rapport (1991) "Statistical Channel Impulse Models for Factory and Open Plan Building Radio Communication System Design" IEEE Transactions on Communications, Vol. COM-39, No. 5,pp794-806.

[17] J. C. Libert and T. S. Rappaport (1996)" A Geometrically Based Model for Line of Sight Multipath Radio Channel" IEEE $46^{\text {th }}$ Vehicular Technology Conference, Atlanta, pp $844-848$.

[18] European cooperation in the Field of Scientific and Technical Research EURO-COST231 (1991) " Urban transmission loss models for mobile radio in the $900 \mathrm{MHz}$ and $1800 \mathrm{MHz}$ bands" Revision 2, The Hague.

[19] Y. Oda, R. Tsuchihashi, K. Tsuenekawa, M. Hata(2001)" Measured Path Loss and Multipath propagation Characteristics in UHF and Microwave Frequency Bands for Urban Mobile Communications" Proc. 53 IEEE Vehic. Technol. Conf. Vol. 1, pp 337-341.

[20] S. Bi, C. K. Ho, and R. Zhang (2015) "Wireless powered communication: Opportunities and challenges," IEEE Commun. Mag.

[21] Beekhuizen et al.( 2014), "Modelling indoor electromagnetic fields (EMF) from mobile phone base stations for epidemiological studies," Elsevier, Environmental International, Vol.67, June, pp $22-26$

[22] Raymond Steele and La jos Hanzo. (2000) “Mobile Radio Communications" $2^{\text {nd }}$ ed., the Electronics and Computer Science Department at the University of Southampton and at Multiple Access Communications Ltd. pp1-181.

[23] K. Sato et al. (1995)" Measurements of Reflection Characteristics and Refractive Indices of Interior Construction

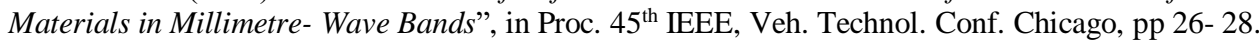

[24] Nathan Blaunstein and Christos Christodoulou (2007)" Radio Propagation and Adaptive Antennas for Wireless Communication Links, Terrestrial, Atomspheric, and Ionospheric" A John Wiley and Sons. pp $81-494$

[25] Firas Mohammed Ali Al-Raie(2010)" Simulation of Multipath Fading Effects in Mobile Radio Systems" Microwave Journal, Online Paper, pp 1-14.

[26] Simon R. Saunders (2007) "Antennas and Propagation for Wireless Communication Systems" $2^{\text {nd }}$ edition, John Wiley and Sons Ltd. pp $25-103$.

[27] Feuerstein, M. J., Blackard, K. L., Rappaport, T. S., Seidel, S. Y. and Xia, H. H., (1994) "Path Loss Delay Spread and Outage Models as Functions of Antenna Height for Microcellular System Design" IEEE Transactions on Vehicular Technology, Vol. 43, No.3, pp 487-498. 
[28] Jorgen Bach. Andersen, Theodore S. Rappaport, and Susumu Yoshida (1995)" Propagation Measurements and Models for Wireless Communication Channel” IEEE COMM. Magazine. pp42 -49.

[29] Molkdar, D. (1991) "Review on Radio Propagation into and within Building " IEEE Proceedings, Vol.138, No.1, pp $61-73$.

[30] Hashemi, H.( 1993) "The Indoor Radio Propagation Channel," Proceedings of IEEE, Vol. 81, No.7, pp, 943-968.

[31] T.K. Sarkar, Z. Ji, K. Kim, A. Medouri, and M. Salazar-Palma, (2003) "A Survey of Various and Propagation Models for Mobile Communication," IEEE Antennas and Propagation Magazine, vol. 45, no. 3, June, pp. 51-82.

[32] M. A. Alim*, M. M. Rahman, M. M. Hossain, and A. Al-Nahid (2010)" Analysis of Large-Scale Propagation Models for Mobile Communications in Urban Area" (IJCSIS) International Journal of Computer Science and Information Security, Vol. 7, No. 1, pp 135 - 139.

[33] Alexander, S. E. (1982)" Radio Propagation Within Building at 900MHz" Electronics Letters, Vol. 18, No. 21, pp 913 - 914.

[34] Durgin G. D and Rappaport T. S. (1998) " A Basic Relationship between Multipath Angular Spread and Narrow Band fading in a Wireless Channel” IEE Electronics Letter, Vol. 34, pp 2431 - 2432.

[35] Durgin G. D and Rappaport T. S. (2000)" Theory of Multipath Shape Factors for Small Scale Fading Wireless Channels" IEEE Transactions on Antennas and Propagation, Vol. 48, No. 5, pp 680 - 693.

[36] J. A. Wepman, J.R. Hoffman, L. H. Loew (1994) “Impulse Response Measurements in the $1850-1990 \mathrm{MHz}$ Band in Large Outdoor Cells" NTIA Report 94 - 309.

[37] Victor Fung, Theodore Rappaport and Berthold Thoma (1993)" Bit Error Simulation for $\frac{\pi}{4}$ DQPSK Mobile Radio Communications "Vol. 11, No 3., pp $393-405$.

[38] Bertoni, H. (2000)"Radio Propagation for Modern Wireless Systems" Upper Saddle River, NJ. Prentice, Hall. pp 141-248.

[39] Thurwachter C. (2000)" Data and Telecommunications: Systems and Applications" Upper Saddle River, NJ., Prentice, Hall.

\section{BIOGRAPHIES OF AUTHORS}

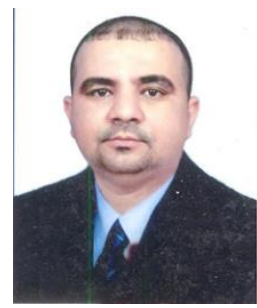

Dr. Jafaar Fahad A.Rida. Received his Bacholar of Electronic and Communication Engineering Technical Najaf Collage in Iraq in 2003. He obtained M. Tech. Communication System Engineering from SHIATS Allahabad India in 2012. He completed Ph.D. in Electronic and Communication Engineering from SHIATS India in 2015. He has experience for five years in CDMA technical company in wireless system and MW system. He has experience in teaching Electronics subject, Advance digital electronic, Digital technology, Data Communication and Computer Networks, and Mobile Cellular Wireless Communication Engineering and biomedical Engineering in Collage of Engineering in Thiqar University. He has research papers about Carbon Nanotube Technology. He is working now teacher in Department of Information System Computer in Collage of Computer Science and Information Technology in Sumer University in Dhiqar in Iraq since December, 2016. No. of Mob. : +9647819366700

E- Mail: j.fahad@uos.edu.iq \& jafaarfahad@gmail.com \& jafaarfahad@yahoo.com 